\title{
ESTUDO DE EQUILÍBRIO DA ADSORÇÃO DE COBRE (II) POR FILMES DE QUITOSANA
}

\author{
M. SARTORI ${ }^{1}$, T. ESPÍNDOLA ${ }^{1}$, J. M. MOURA ${ }^{1}$, N. SILVEIRA Jr ${ }^{1}$. T. R. CADAVAL Jr ${ }^{1}$, \\ L. A. A. PINTO ${ }^{1}$ \\ ${ }^{1}$ Universidade Federal do Rio Grande, Escola de Química e Alimentos \\ E-mail para contato: mariliasartori09@hotmail.com
}

\begin{abstract}
RESUMO - Neste trabalho, o efeito da temperatura foi estudado na adsorção de íons cobre utilizando filmes de quitosana como adsorvente. A fim de representar o comportamento no equilíbrio, foram ajustados os modelos de isotermas de Henry, e Freundlich para caracterizar a relação adsorvente-adsorbato em diferentes temperaturas. O modelo mais adequado para representar as isotermas de equilíbrio para a adsorção de cobre por filmes de quitosana foi o de Freundlich apresentado o menor valor de erro médio relativo (EMR) no ajuste dos dados.
\end{abstract}

\section{INTRODUÇÃO}

A poluição de águas subterrâneas e superficiais por efluentes provenientes de processos químicos é um problema de escala mundial. A presença de metais pesados em mananciais advindos destes descartes causam uma série de transtornos pois estes metais tendem a serem cumulativos em organismos vivos, causando várias doenças e distúrbios (Ghaee et al., 2010). Por outro lado, estes metais desempenham papéis importantes na maioria das indústrias, principalmente nos setores de mineração, galvanoplastia, farmácia, fabricação de dispositivos elétricos e pesticidas (Igberase et al., 2014). A poluição por cobre é particularmente preocupante, pois ao mesmo tempo em que desempenha um papel essencial no metabolismo animal, é tóxico em concentrações elevadas, com efeitos que incluem vômitos, cólicas, convulsões e até mesmo a morte (Ghaee et al., 2010). Segundo a resolução do Conselho Nacional do Meio Ambiente o limite máximo de lançamento de cobre nos corpos hídricos é de $1,0 \mathrm{mg} \mathrm{L}^{-1}$, todavia nos processos industriais onde é utilizado o cobre, esse valor é superior, havendo a necessidade de se realizar um tratamento prévio destes efluentes antes do descarte.

Assim, vários métodos têm sido utilizados para remoção de íons metálicos a partir de efluentes industriais. Dentre os métodos convencionais estão à precipitação química, separação por membrana, troca iônica, evaporação, eletrólise, etc. Porém na maioria das vezes esses processos são complexos e ineficazes, principalmente quando esses íons estão presentes em baixas concentrações (Li e Bai, 2005). Nesse contexto a adsorção se apresenta como uma técnica promissora na remoção desses íons devido as facilidade de operação, simplicidade e relação custo benefício (Igberase et al., 2014). Sendo assim há uma busca crescente por adsorventes obtidos de fontes renováveis, dentre os quais se destaca a quitosana (Mi et al., 2015). 
A quitosana, poli $(\beta-1 \rightarrow 4)-2-$ amino-2-deoxi-Dglucopiranose, é produzida através da desacetilação parcial da quitina, que é amplamente encontrada em exoesqueletos de crustáceos e na parede celular de fungos (Ngah et al., 2011). Além das várias propriedades que a quitosana apresenta, como biocompatibilidade, biodegradabilidade, não toxicidade, etc., esta também é conhecida como um excelente adsorvente para íons de metais de transição, pois possui vários grupos reativos que interagem com os íons metálicos através de vários mecanismos, que dependem do íon, do $\mathrm{pH}$ e da composição da solução (Yu et al., 2013). Os grupos amina $\left(-\mathrm{NH}_{2}\right)$ da quitosana são reconhecidos como os principais grupos ativos para interação com íons metálicos, enquanto que os grupos hidroxila $(\mathrm{OH})$, especialmente na posição C-3, também podem contribuir no processo de adsorção (Zhao et al., 2007).

Além de todas as características já citadas, a quitosana pode ser preparada em diferentes formas, tais como pó, nanopartículas, hidrogéis, membranas, esponjas e fibras. No entanto a separação do material, após a adsorção é bastante difícil, havendo a necessidade de subsequentes processos de separação (Mirmohseni et al., 2012). Sendo assim, esta dificuldade leva a necessidade do desenvolvimento de materiais à base de quitosana, que facilitem a separação do adsorvente após a operação de adsorção, como é o caso do polímero na forma de filme. Tais filmes, além de manterem as propriedades de adsorção do polímero, facilitam a remoção do adsorvente após a operação e apresentam boas propriedades mecânicas que facilitam a sua reutilização (Dotto et al., 2013).

No entanto para a utilização de filmes de quitosana na adsorção de íons cobre (II), são necessários estudos para uma melhor compreensão e entendimento do processo. O estudo das isotermas é fundamental, uma vez que a mesma auxilia no esclarecimento do comportamento da operação em função da temperatura bem como em termos de equilíbrio. Além disso, os parâmetros de equilíbrio fornecem informações sobre a qualidade do adsorvente e a afinidade adsorvente-adsorbato (Dotto et al., 2013). Sendo assim, o objetivo deste trabalho foi estudar as isotermas de adsorção de íons cobre (II) por filmes de quitosana. Foi verificado o efeito da temperatura e os modelos de Henry e Freundlich foram ajustados aos dados experimentais.

\section{MATERIAIS E MÉTODOS}

\subsection{Preparação do Filme de Quitosana}

A quitosana (massa molar $150 \pm 3 \mathrm{kDa}$ e grau de desacetilação $85 \pm 1 \%$ ) foi obtida a partir de resíduos de camarão (Penaeus brasiliensis) segundo Weska et al. (2007) . Os filmes foram produzidos utilizando a técnica casting de acordo com trabalhos anteriores. Os valores de tensão de ruptura e alongamento dos filmes foram determinados utilizando um texturômetro (Stable Micro Systems, TA-XT-2i, Reino Unido) com uma carga de $50 \mathrm{~N}$ e velocidade de elongação de $2 \mathrm{~mm} \mathrm{~s}^{-1}$. A espessura dos filmes foi mensurada utilizando um micrômetro digital (Insize, IP54, Brasil) com resolução de 0,0010 $\mu \mathrm{m}$.

\subsection{Experimentos de Adsorção}

Primeiramente, foi preparada uma solução estoque com concentração de $1,00 \mathrm{~g} \mathrm{~L}^{-1} \mathrm{de}$ cobre a partir da dissolução de $\mathrm{CuSO}_{4} \cdot 5 \mathrm{H}_{2} \mathrm{O}$. As diluições subsequentes foram realizadas com água destilada, a partir da solução estoque e o pH ajustado com tampão fosfato 
dissódico/ácido cítrico à pH 5,0 (DOTTO et al., 2013). Os experimentos foram realizados em triplicata $(n=3)$, e todos os reagentes foram de grau analítico.

Para os experimentos de adsorção, filmes em concentração de $250 \mathrm{mg} . \mathrm{L}^{-1}$, foram adicionados em soluções de cobre com concentrações iniciais entre 25-200 mg. $\mathrm{L}^{-1}$. Os ensaios foram realizados em um agitador do tipo Wagner com temperaturas de $25^{\circ} \mathrm{C}, 35^{\circ} \mathrm{C}, 45^{\circ} \mathrm{C}$ e $55^{\circ} \mathrm{C}$, sob uma taxa de agitação de $100 \mathrm{rpm}$ até o equilíbrio. Após, as concentrações remanescentes de cobre foram determinadas por espectroscopia de absorção atômica. As capacidades de adsorção nos equilíbrios foram determinadas pela Equação (1).

$$
\mathrm{q}_{\mathrm{e}}=\frac{\mathrm{V}\left(\mathrm{C}_{0}-\mathrm{C}_{\mathrm{e}}\right)}{\mathrm{m}}
$$

Sendo $\mathrm{C}_{0}$ e $\mathrm{C}_{\mathrm{e}}$, respectivamente, as concentrações inicial e de equilíbrio na fase líquida (mg.L $\left.\mathrm{L}^{-1}\right), \mathrm{m}$ a massa de adsorvente (g) e V o volume da solução (L).

\subsection{Estudo do equilíbrio}

Modelos de adsorção isotérmica são amplamente utilizados para descrever processos de adsorção e investigar o equilíbrio da adsorção (DOTTO et al., 2013). Neste trabalho, dados de equilíbrio foram obtidos em $25,35,45$ e $55^{\circ} \mathrm{C}$ e as curvas foram ajustadas aos modelos isotérmicos de Henry e Freundlich.

A isoterma de Henry é utilizada quando a relação entre a quantidade de adsorbato na fase fluida e a quantidade de adsorbato na fase sólida é linear, com uma constante de proporcionalidade chamada de constante de equilíbrio de Henry $\left(\mathrm{K}_{\mathrm{H}}\right)$ e pode ser representada pela Equação 2:

$$
\mathrm{q}_{\mathrm{e}}=\mathrm{k}_{\mathrm{H}} \mathrm{C}_{e}
$$

sendo, $\mathrm{q}_{\mathrm{e}}$ a capacidade de adsorção no equilíbrio $\left(\mathrm{mg} \mathrm{g}^{-1}\right)$, e $\mathrm{C}_{\mathrm{e}}$ a concentração da fase líquida no equilíbrio ( $\left.\mathrm{mg} \mathrm{L}^{-1}\right)$ e $\mathrm{k}_{\mathrm{H}}$ é a constante de Henry .

O modelo de Freundlich assume que a superfície de adsorção é heterogênea e pode ser representado pela Equação 3.

$$
\mathrm{q}_{\mathrm{e}}=\mathrm{k}_{\mathrm{F}} \mathrm{C}_{\mathrm{e}}^{1 / \mathrm{n}}
$$

onde, $\mathrm{k}_{\mathrm{F}}$ é a constante de Freundlich $\left(\left(\mathrm{mg} \mathrm{g}^{-1}\right)\left(\mathrm{mg} \mathrm{L}^{-1}\right)^{-1 / n}\right)$ e $1 / n$ é o fator de heterogeneidade

Os parâmetros de equilíbrio foram determinados por regressão não linear, usando Statistic 7.0 (Statsoft, USA). Os erros relativos médios (EMR) foram usados para selecionar o melhor modelo isotérmico. 


\section{RESULTADOS E DISCUSSÃO}

\subsection{Caracterização dos filmes de quitosana}

Foi observado que o filme apresentou tensão de ruptura de $22,1 \pm 1,1 \mathrm{MPa}$ e alongamento de $10,5 \pm 2,1 \%$. Estas propriedades se mantiveram após a adsorção e a espessura do filme foi de $68 \pm 2 \mu \mathrm{m}$.

\subsection{Isotermas de adsorção}

A Figura 1 apresenta os dados experimentais de equilíbrio para adsorção de íons de cobre por filme de quitosana.

Figura 1 - Isotermas de equilíbrio para o sistema $\mathrm{Cu}^{2+}$ - Filmes de quitosana.

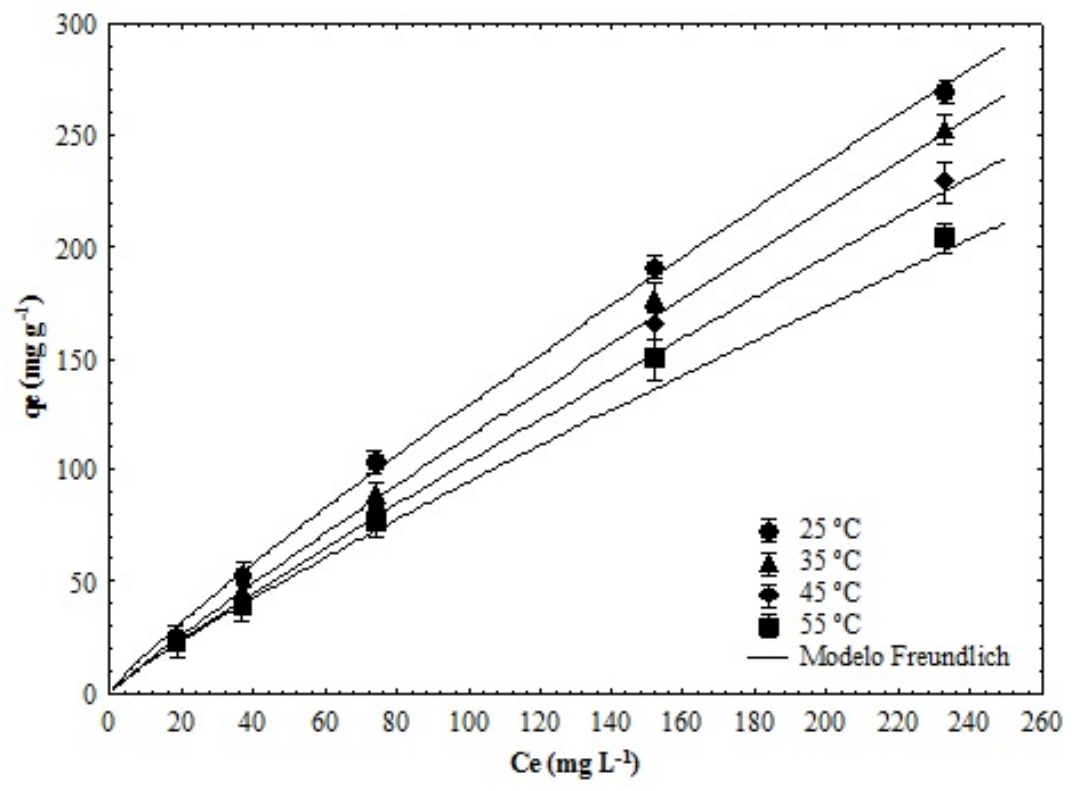

Tabela 1 - Propriedades dos sistemas (fluido-partícula).

\begin{tabular}{ccccc}
\hline \multirow{2}{*}{$\mathrm{T}(\mathrm{K})$} & Henry & & & \\
\cline { 2 - 5 } & $\mathrm{K}_{\mathrm{H}}\left(\mathrm{L} \mathrm{g}^{-1}\right)$ & & $\mathrm{R}^{2}$ & EMR (\%) \\
\hline 298 & 1,2044 & & 0,9905 & 8,91 \\
308 & 1,0958 & & 0,9962 & 5,65 \\
318 & 0,9824 & & 0,9935 & 7,34 \\
328 & 0,8711 & & 0,9881 & 10,45 \\
\hline $\mathrm{T}(\mathrm{K})$ & Freundlich & $\mathrm{n}_{\mathrm{F}}$ & $\mathrm{R}^{2}$ & $\mathrm{EMR}(\%)$ \\
\cline { 2 - 5 } & $\mathrm{k}_{\mathrm{F}}\left(\mathrm{mg} \mathrm{g}^{-1}\right)\left(\mathrm{mg} \mathrm{L}^{-1}\right)^{-1 / \mathrm{n}_{\mathrm{F}}}$ & 1,14 & 0,9987 & 5,43 \\
298 & 2,2542 & 1,08 & 0,9988 & 3,18 \\
308 & 1,5918 & 1,09 & 0,9971 & 3,68 \\
318 & 1,5182 & 1,14 & 0,9965 & 4,29 \\
328 & 1,6509 & & & \\
\hline
\end{tabular}


Baseado nos resultados da Tabela, ( $\mathrm{R}^{2}$ e EMR) é possível afirmar que o modelo de Freundlich é o mais apropriado para ajustar os dados experimentais de equilíbrio entre os íons de cobre e o filme de quitosana.

\section{CONCLUSÃO}

Neste trabalho, a remoção de cobre a partir de soluções aquosas, por adsorção, utilizando filmes de quitosana foi estudada. O modelo de Freundlich foi o mais adequado para representar as isotermas de equilíbrio de adsorção com maior $\mathrm{R}^{2}$ e menor EMR.

\section{REFERÊNCIAS}

DOTTO, G. L., MOURA, J. M., CADAVAL, T. R. S., PINTO, L. A. A. Application of chitosan films for the removal of food dyes from aqueous solutions by adsorption. Chem. Eng. J., v. 214, p. 8-16, 2013.

GHAEE, A.; SHARIATY-NIASSAR, M.; BARZIN, J.; MATSUURA, T. Effects of chitosan membrane morphology on copper ion adsorption. Chem. Eng. J., v. 165, p. 46-55, 2010.

IGBERASE, E.; OSIFO, P.; OFOMAJA, A. The adsorption of copper (II) ions by polyaniline graft chitosan beads from aqueous solution: Equilibrium, kinetic and desorption studies. J. Environ. Chem. Eng. J., v. 2, p. 362-369, 2014.

JIANG, X., CHEN, L., ZHONG, W. I. A new linear potentiometric titration method for the determination of deacetylation degree of chitosan. Carbohydr. Polym., 54, 457-463, 2003.

LI, N.; BAI, R. Copper adsorption on chitosan-cellulose hydrogel beads: behaviors and mechanisms. Sep. Purif. Technol., v. 42, p. 237-247, 2005.

MI, F.; WU, S. J.; LIN, F. Adsorption of copper(II) ions by a chitosan-oxalate complex biosorbent. Int. J. Biol. Macromol., v. 72, p. 136-144, 2015.

MIRMOHSENI, A.; DORRAJI, M. S. S.; FIGOLI, A.; TASSELLI, F. Chitosan hollow fibers as effective biosorbent toward dye: Preparation and modeling. Bioresour. Technol., v. 121, p. 212-220, 2012.

NGAH, W. S. W.; TEONG, L. C.; HANAFIAH, T. M. A. K. M. Adsorption of dyes and heavy metal ions by chitosan composites: A review. Carbohydr. Polym., v. 83, p. 1446-1456, 2011.

WESKA, R. F.; MOURA, J. M.; BATISTA, L. M.,; RIZZI, J.; PINTO, L. A. A. Optimization of deacetylation in the production of chitosan from shrimp wastes: Use of response surface methodology. J. Food Eng., v. 80, p. 749-753, 2007.

YU, K.; HO, J.; MCCANDLISH, E.; BUCKLEY, B.; PATEL, R.; LI, Z.; SHAPLEY, N. C. Copper ion adsorption by chitosan nanoparticles and alginate microparticles for water purification applications. Colloids Surf., A, v. 425, p. 31-41, 2013. 
ZHAO, F.; YU, B.; YUE, Z.; WANG, T.; WEN, X.; LIU, Z.; ZHAO, C. Preparation of porous chitosan gel beads for copper(II) ion adsorption. J. Hazard. Mater., v. 147, p. 67-73, 2007. 